\title{
User Intention Driven Adaptive Gait Assistance using a Wearable Exoskeleton
}

\author{
Vijaykumar Rajasekaran $^{1 *}$, Joan Aranda ${ }^{1,2}$ and Alicia Casals ${ }^{1,2}$ \\ ${ }^{1}$ Universitat Politécnica de Catalunya, Barcelona-Tech, Spain \\ ${ }^{2}$ Institute for Bioengineering of Catalonia, Barcelona, Spain
}

\begin{abstract}
A user intention based rehabilitation strategy for a lower-limb wearable robot is proposed and evaluated. The control strategy, which involves monitoring the human-orthosis interaction torques, determines the gait initiation instant and modifies orthosis operation for gait assistance, when needed. Orthosis operation is classified as assistive or resistive in function of its evolution with respect to a normal gait pattern. The control algorithm relies on the adaptation of the joints' stiffness in function of their interaction torques and their deviation from the desired trajectories. An average of recorded gaits obtained from healthy subjects is used as reference input. The objective of this work is to develop a control strategy that can trigger the gait initiation from the user's intention and maintain the dynamic stability, using an efficient real-time stiffness adaptation for multiple joints, simultaneously maintaining their synchronization. The algorithm has been tested with five healthy subjects showing its efficient behavior in initiating the gait and maintaining the equilibrium while walking in presence of external forces. The work is performed as a preliminary study to assist patients suffering from incomplete Spinal cord injury and Stroke.
\end{abstract}

Keywords - Adaptive control, Exoskeleton, Gait initiation, Gait assistance, Wearable robot

\section{Introduction}

Human centered rehabilitation is essential for ensuring the user involvement in a therapy. Several human centered strategies, such as patient cooperative and support motor function assessment, oriented to the development of robot behaviors have been widely studied [1]. These strategies support the assist-as-needed concept by determining the level of robotic assistance provided to the user. Referring to the gait, assistance must be dynamically adapted to the patient's needs and thus, it is necessary to develop a personalized assistance in function of the user intentions and movements, as well as the therapy, which involves the knowledge of assistance to be perceived. Other factors have a direct influence on the quality of assistance, such as the availability of mechanical support, control strategies, combination of assistive devices etc. One of the widely used approaches to detect and evaluate the need of assistance is by evaluating the position errors. However, the use of a predefined trajectory pattern, without other inputs, imposes a complete assistance which might induce slacking and harm 
the patient. Thus, it is necessary to measure the human-orthosis interaction torques, to evaluate the user performance and status, to design a hybrid combination of forceposition control. Assistance in robotic rehabilitation can be achieved using an effective control strategy [2] such as impedance or adaptive control, which act based on the subject's performance. Such control strategies operate under the principle of assistance-as-needed, in which assistive forces increase as the participant deviates from the desired trajectory. This deviation can also be used as input to generate a trigger to initiate the movement or the assistance in accordance to the user's performance.

The detection of the best instant for gait initiation and termination has been studied by many researchers in order to design a volitional control based robotic rehabilitation $[3,4]$. One of the widely used approaches for monitoring the human intention relies on the use of brain machine interfaces such as in XoR [5]. These systems are efficient in monitoring the user intention, because a real displacement of the joint position is not needed to initiate the gait. Instead, gait initiation in MINDWALKER is based on the displacement of the CoM (center of mass) which is calculated heuristically [6]. HAL (Hybrid Assistive limb), a light weight powered exoskeleton suit, is efficient in adapting to the user movements by sensing the muscle synergies. HAL uses myoelectric signals to measure the muscle forces and to support voluntary motion of the patient $[7,8]$, as well as to determine the joint stiffness to be applied.

Gait assistance using wearable robots is challenging in terms of determining the suitable assistance for dynamic stability, considering the ground reaction forces. Several wearable robots have succeeded in providing dynamic stability, such as BLEEX [9], XPED2 [10], Ekso (earlier eLegs) [11], Rex (Rex Bionics) and Re-Walk [12], and have proven to be efficient in providing assistance on a passive range of motion and using complex systems.

The objective of this work is to develop an assistive control strategy for a wearable robot to perform user-dependent gait initiation and assistance. The gait initiation approach ensures the user involvement in the therapy and their motivation in performing the task. The control strategy implies the implementation of an impedance based approach for gait assistance, without using neither treadmill nor body weight support. The absence of weight compensation carries with it the challenge of maintaining the equilibrium in presence of ground reaction forces. The goal is to develop an efficient control model for a low-cost wearable robot and to validate the assistive behavior of the robot for patients with neurological disorders. A hybrid position and interaction torques based control strategy is presented to continuously adapt the user movements to the desired gait pattern in real time. This real time adaptation also ensures synchronization among the joint trajectories to maintain dynamic stability.

\section{$2 \quad$ Human Centered Gait}

The human-orthosis interaction torques are essential in defining the dynamic analysis of a human centered control strategy. Hence, the mathematical model for the dynamic analysis of an exoskeleton can be represented as

$$
M_{\text {ort }}(q) \ddot{q}+C_{\text {ort }}(q, \dot{q})+G_{\text {ort }}(q)=\tau_{a}+\tau_{\text {pat }}+\tau_{d}
$$


where $q, \dot{q}, \ddot{q}$ are the vectors of joint positions, velocities and accelerations. $M_{\text {ort }}(q)$ is the inertia matrix, $C_{\text {ort }}(q, \dot{q})$ is the centrifugal and Coriolis vector and $G_{\text {ort }}(q)$ represents the gravitational torques. $\tau_{a}$ and $\tau_{\text {pat }}$ are the orthosis and patient torques respectively and $\tau_{d}$ corresponds to the external disturbances acting on the subject. These actuator and patient torques are influenced by the human-orthosis interaction, while the external disturbances can be due to any assistive or external sources which can affect the dynamic stability of the robot. In the present work, $\tau_{\text {pat }}$ is used for gait initiation and to determine the level of assistance to be exerted by the orthosis.

\subsection{Gait Initiation}

The gait initiation can be defined as the time $t$ in which the user intends to perform a movement and can be determined from the human-orthosis interaction torques. This type of user dependent initiation is efficient in influencing or motivating the user to provide an input movement. The user motivation is necessary to improve the therapy and avoid slacking. Since human-orthosis interaction torques based gait initiation can be a drawback in differentiating between a tremor and the user intention, the joint position and torques must be considered too to trigger the therapeutic procedure. Additionally, this strategy permits the user to initiate the therapy with the leg he or she feels more comfortable, fig 1.

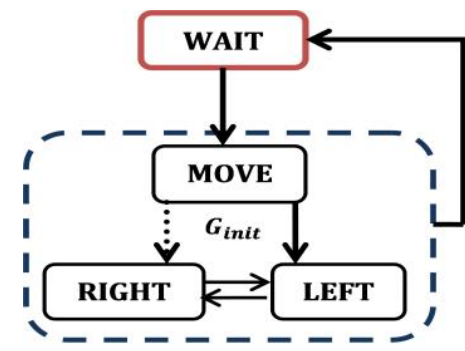

Fig. 1. State transition of the gait initiation algorithm

The gait initiation strategy considers the joint positions and the human-orthosis interaction torques of both legs. This can be represented as,

$$
G_{\text {init }}=\operatorname{Leg}_{x}, x \in[R, L]
$$

where, $G_{\text {init }}$ is the gait initiation trigger obtained from the user for $\operatorname{Leg}_{x} . \theta_{R}$ and $\theta_{L}$ are the joint angles of right and left leg respectively; $\tau_{R}$ and $\tau_{L}$ corresponds to the measured interaction torques in right and left leg respectively.

$$
\begin{aligned}
& \begin{array}{l}
\text { Algorithm 1: Gait initiation } \\
\text { Case } \theta_{R}(t) \neq \theta_{R}(t-1), \tau_{R}
\end{array}(t) \neq \tau_{R}(t-1) \& \& \theta_{L}(t)=\theta_{L}(t-1) \\
& \quad x=R \\
& \text { Else } x=L
\end{aligned}
$$




\subsection{Gait Assistance}

In the present work, for each joint, the actuator works in collaboration with the patient. The actuator torque can be modified by varying the joint stiffness parameter, which invariably modifies the corresponding joint trajectory and force compensation. This stiffness variation alters the actuator torque which determines the degree of control transferred from the orthosis to the human or vice versa. Such an impedance control scheme has been widely used for its compliant behavior, which results in an adaptive walking pattern and a more natural interaction between patient and orthosis. Thus the impedance control can be determined by equation (3)

$$
F=M a+C v+K \cdot\left(\theta_{\text {ref }}-\theta_{\text {act }}\right)
$$

where, $\theta_{\text {ref }}$ and $\theta_{\text {act }}$ are the reference and actual joint positions respectively, $\mathrm{K}$ is the stiffness parameter of the joint and $\mathrm{F}$ represents the applied force to the joint. $\mathrm{M}$ represents the mass, $\mathrm{C}$ is the damping constant and $a$ and $v$ represent the acceleration and velocity of the robot. Here, the input sample rate to the system is maintained constant and the damping coefficient is kept small, therefore the velocity of the orthosis is not modified by the user and thus, it does not induce any significant effect on the applied force. Hence the force equation, influenced by the position error, is modified as:

$$
F=K\left(\theta_{\text {ref }}-\theta_{\text {act }}\right)
$$

The value $\mathrm{K}$ can be determined dynamically based on the performance of the user and the level of assistance to be exerted by the orthosis.

$$
\begin{gathered}
K_{t+1}=K_{t} \pm \Delta K \\
\Delta K=\left|\left(\frac{\theta_{\text {ref }}-\theta_{a c t}}{s^{*} \tau_{\text {pat }}}\right)\right|
\end{gathered}
$$

where, $s$ is a confidence factor which is used to determine the stiffness value at time $t+1$. The confidence factor is a variable which shall be defined by the therapist in function of the capabilities of the patient. This confidence factor can be varied according to the progress of the user, in order to modify the time instant at which assistance

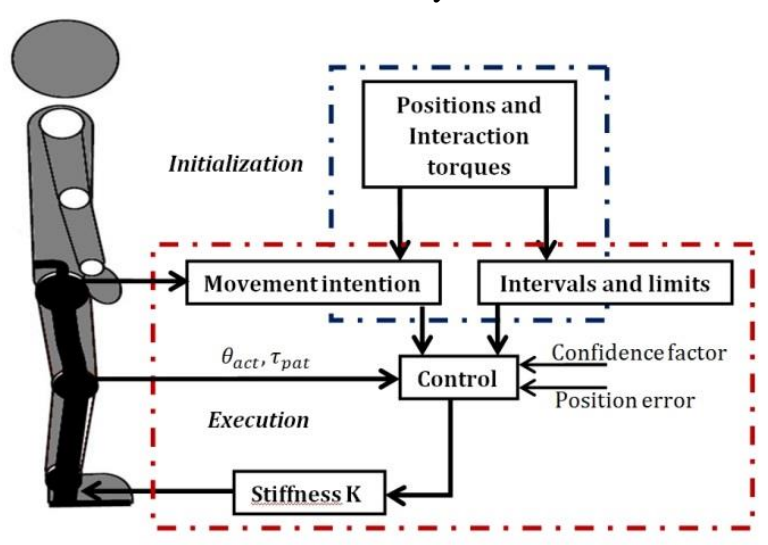

Fig. 2. Schematic representation of the user-intention based adaptive control strategy 
is to be initiated. A low confidence factor means that the level of assistance should be high and a higher confidence factor indicates that the subject is capable of walking without or with little assistance. A variation of the $\mathrm{K}$ value results in a change in the force acting at the joint level what is perceived as assistance or resistance by the patient.

The stiffness variation module, as shown in fig 2, is responsible for incrementing, decrementing or maintaining the stiffness parameter of each joint. Within a given range of trajectory errors, stiffness is computed from position errors, but when the defined error thresholds are surpassed, stiffness should be modified according to the measured interaction torques. This condition takes place in general with the presence of external perturbations. The following are the parameters involved in defining this stiffness variation:

$\theta_{\mathrm{e}}$ - Position error $(\mathrm{deg})$

$\theta_{\mathrm{e}} \mathrm{Th}_{\mathrm{up}}-$ Upper threshold of position error $(\mathrm{deg})$

$\theta_{\mathrm{e}} \mathrm{Th}_{\mathrm{lo}}-$ Lower threshold of position error $(\mathrm{deg})$

$\tau_{\text {pat }}$ - Human-orthosis interaction torques $(\mathrm{Nm})$

$\tau_{\text {pat }} \mathrm{Th}_{\mathrm{lo}}$ - Lower threshold of interaction torques $(\mathrm{Nm})$

$\tau_{\text {pat }} \mathrm{Th}_{\mathrm{up}}$ - Upper threshold of interaction torques $(\mathrm{Nm})$

$\Delta \mathrm{K}$ - Stiffness variation $(N / m)$

Algorithm 2: Gait assistance
$\begin{aligned} & \text { Case } \theta_{\mathrm{e}}>\theta_{\mathrm{e}} \mathrm{Th}_{\mathrm{up}} \\ & \mathrm{K}=\mathrm{K}+\Delta \mathrm{K} \\ & \text { Case } \theta_{\mathrm{e}}<\theta_{\mathrm{e}} \mathrm{Th}_{\mathrm{lo}} \& \& \tau_{\mathrm{pat}} \mathrm{Th}_{\mathrm{lo}}<\tau_{\text {pat }}<\tau_{\text {pat }} \mathrm{Th}_{\mathrm{up}} \\ & \mathrm{K}=\mathrm{K}-\Delta \mathrm{K} \\ & \text { Else } \\ & \text { Maintain } K\end{aligned}$

An average walking pattern generated from a set of recorded walking patterns of healthy individuals is used as reference input. Based on this pattern and the patient contribution, the stiffness $\mathrm{K}$ establishes the operating mode at each joint, assistive or resistive. In a first set of trials, for each subject the maximum interaction torques are obtained by applying a low stiffness value at each joint. From these maxima, we extract the upper and lower thresholds of interaction torques to dynamically define the operation mode. These thresholds are obtained by multiplying these maxima interaction torques by the confidence factor $(s)$.

\section{Experimental Procedure}

The proposed intention driven adaptive strategy is based on the position error and interaction torques. The strategy needs an initial study about the user adaptation to determine the gait initiation and assistance scenario. Hence, the experimentation consists of two phases: initialization and execution. The initialization phase involves monitoring the interaction torques and joint positions with no-assistance provided by 
the orthosis in order to be able to define $s$. This initialization phase is used to parameterize the user intentions and adaptations to the movement. In the execution phase, the changes in movement and interaction torques are used as a trigger for gait initiation. The interaction torques, limited by the confidence factor, are used to determine the time instants of stiffness variation. Both these phases are performed and evaluated using a lower-limb exoskeleton.

\subsection{Exoskeleton}

H1 is a 6 DoF (degree of freedom) wearable lower limb orthosis with an anthropomorphic configuration to assist individuals with incomplete Spinal cord injury (SCI) or Stroke. The exoskeleton, shown in fig 3, has been built within the framework of the Hyper* project. H1 has three joints for each leg: hip, knee and ankle, each joint is powered by a DC motor coupled with a harmonic drive gear. The exoskeleton is equipped with potentiometers and strain gauges to measure the joint angles and human-orthosis interaction torques on the links respectively. A detailed description about the exoskeleton structure and communication parameters is detailed in [13]. The variable stiffness control ensures a safe therapeutic experience [14, 15]. The exoskeleton permits a stiffness value within the range of $1-100 \mathrm{~N} / \mathrm{m}$. A low stiffness value $(<10 \mathrm{~N} / \mathrm{m})$ will not cause any significant effect on the user's behavior. Similarly, a high stiffness value $(>80 \mathrm{~N} / \mathrm{m})$ will provide a completely assisted movement, with few or no input from the user. The initial stiffness value must be defined taking into account the user's capability and the degree of assistance to be applied by the orthosis.

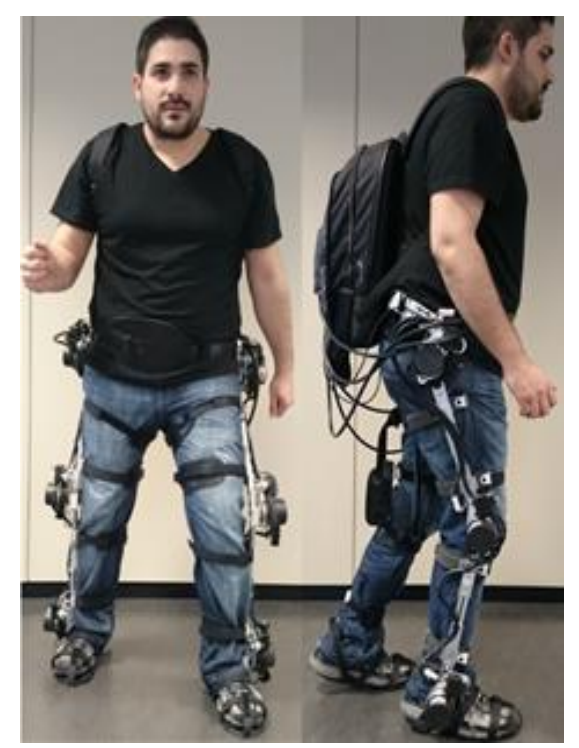

Fig. 3. Participant wearing the HYPER exoskeleton H1 


\subsection{Experimentation}

Initially the walking pattern is generated from tests on subjects applying a low stiffness value $(20 \mathrm{~N} / \mathrm{m})$. This phase is necessary to obtain the pattern of interaction torques and to allow the user to adapt to the orthosis. This initialization also determines the minimum interaction torque observed in the users along with the deviation in position. The evolution of these interaction torques are used to determine the initiation of stiffness variation, determining the adaptive behavior to be exerted by modifying stiffness. This variable stiffness results in either an assistive or resistive behavior. The confidence factor is used to determine the initiating time of the gradual actuation of the joints stiffness functions, thusachieving a smooth performance without affecting the joints trajectories. The gradual increase or decrease of the stiffness value smoothens the interaction torques.

The setup includes a recorded gait pattern obtained from healthy users and optimized after some repetitions of gait cycles. The values of stiffness and confidence factor are defined based on the subject's health condition. Since the strategy is tested with healthy individuals, the initial stiffness and confidence factor are assumed to be $50 \mathrm{~N} / \mathrm{m}$ and 0.9 respectively. High interaction torques are found in healthy subjects, so a higher confidence factor is needed to define their thresholds.

The evaluation of the control strategy is performed by following a protocol and considering an intermediate pause between consecutive trials to allow identifying the user intention after recovering from fatigue. An interval of 10-20 seconds is introduced at the beginning of each trial, for gait initiation algorithm, with an auditory cue to notify the subject to initiate the movement. Similarly for evaluating the gait assistance strategy pause time of 1-2 minutes is considered at the end of every 10 minutes walking test. Thus the study involves two types of walking experiments, gait initiation for 3 minutes and gait assistance for 10 minutes. The gait assistance experiment is performed for 30 minutes, i.e. 3 sets of 10 minutes walking test.

\section{$4 \quad$ Results and Discussion}

The proposed adaptive control strategy has been tested and evaluated with five healthy individuals of the age group $37 \pm 9$, weight $80 \pm 8 \mathrm{~kg}$, and height $1.75 \pm 0.05 \mathrm{~m}$. The results section has been divided into two parts in order to explain the gait initiation and the gait assistance scenarios.

\subsection{Gait Initiation}

In a gait cycle, the knee joint plays a key role for both the initialization of the movement and the swing state. Hence, the gait initiation strategy is evaluated by monitoring the deviation in the knee joint movement with respect to the expected pattern, along with the interaction torques. The flexion and extension movement of the knee joint is monitored to differentiate between the user's intention and tremor movement. As shown in fig 4, the right leg of the user showed gait movement initiation in most of the trials. This can be seen from the shift in the interaction torques of the right and 
left leg, both in hip and knee joint. The initiation of the gait is characterized by the flexion movement of the knee joint. For instance, at time 97 seconds the right knee joint shows a little displacement in the movement which initiates the gait cycle. The hip joint trajectory appears after a few seconds, immediately followed by the transition to the left leg.
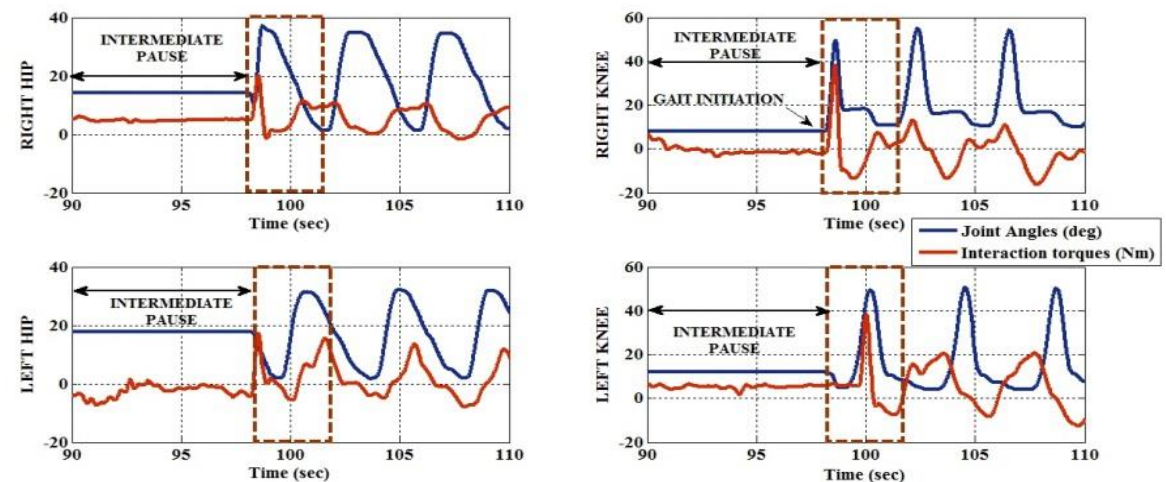

Fig. 4. Gait initiation sequence of a healthy user, blue curve signifies the joint trajectories and red curve indicates the interaction torques

\subsection{Gait Assistance}

The efficiency of the adaptive gait assistance provided by the control model is evaluated in comparison with a reference gait pattern. Fig 5 shows the reference gait patterns and the resulting mean gait cycles of the five healthy subjects. The subjects performed a free normal walking movement, with low stiffness. In this case, the deviation from the desired trajectory was found to be high. After a series of trials (10) this error decreased gradually due to the effect of the adaptive stiffness acting on the joints. The stiffness variation helped to maintain this error within a specified range
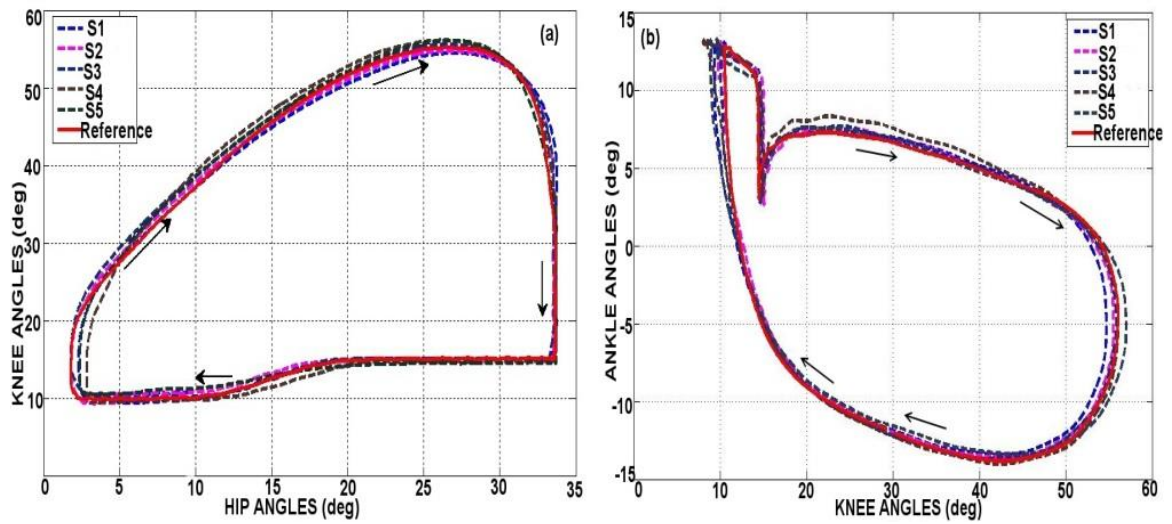

Fig. 5. Reference gait pattern and the resulting mean gait pattern of each subject: (a) Hip (deg) - Knee (deg), (b) Knee (deg) - Ankle (deg) 
and following a similar pattern of incrementing and decrementing $\mathrm{K}$ at every joint, thus resulting in an assistive or resistive behavior.
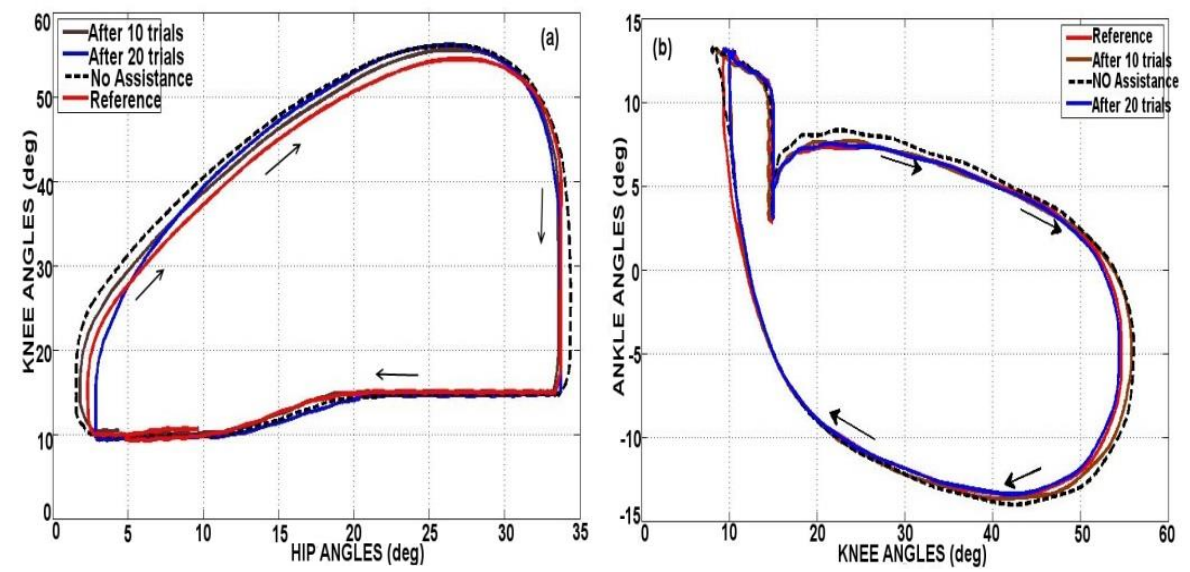

Fig. 7. Changes in gait pattern of a healthy subject due to effect of adaptive stiffness: (a) Hip (deg) - Knee (deg), (b) Knee (deg) - Ankle (deg)

The results of one of the subjects are used to show the response of the control strategy. The gait performance of a healthy user, as shown in fig 6, demonstrates the influence of the stiffness variation proposed in this work. The initial walking with low stiffness value is presented as the 'no-assistance' mode. In comparison with the reference pattern, the no assisted walking is found to produce a maximum deviation. After the application of a variable stiffness, the user is able to walk within a predefined error limits. The stiffness variation also converges with respect to the movement at the end of 10 trials. At the end of 20 trials, the user is following a movement which is quite similar to the reference pattern.

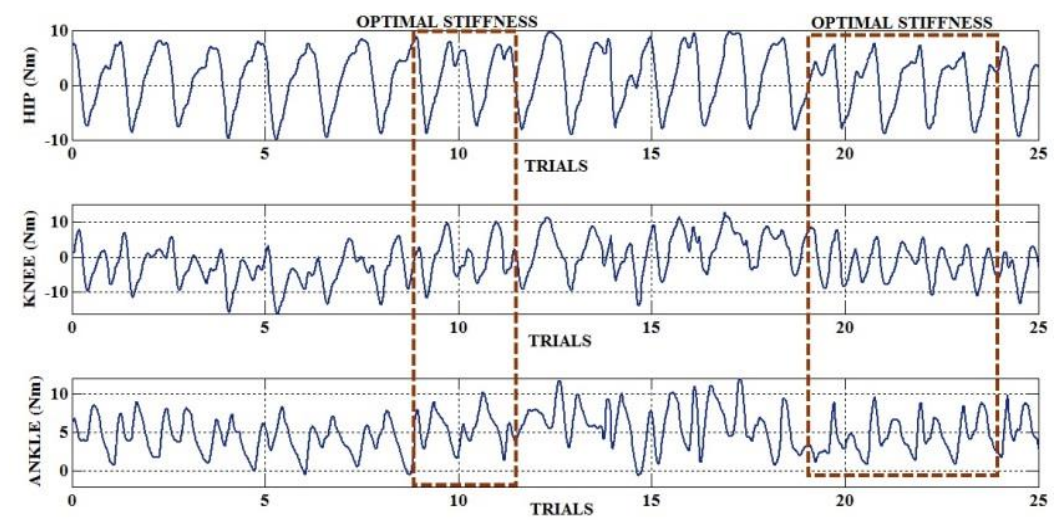

Fig. 6. Interaction torques of each joint showing the change in behavior while stiffness converges (highlighted region) to an optimal value 
The error was found to be within the defined limits for all the users. The hip joint showed a little variation and more adaptable behavior in terms of stiffness changes in real time. Since the exoskeleton is a planar robot, the lateral hip movement cannot be monitored. However, this orthosis limitation does not affect the proposed control strategy. A significant variation of the stiffness is found in both ankle and knee joints. The hip joint stiffness varied in a short range which is evident from the interaction torques in fig 7. This can be due to the lateral movement of the user's hip joint which compensates the joint trajectory. The interaction torques of the ankle joint is in the limits of $12 \mathrm{Nm}$ to $-3 \mathrm{Nm}$, as shown in fig 7, and with the application of the confidence factors the threshold is limited to $10 \mathrm{Nm}$ to $-1 \mathrm{Nm}$. This threshold limit is used to initiate the stiffness increment when the position error threshold is reached. Similarly, in the knee joint, the interaction torques are within the limits of $14 \mathrm{Nm}$ to -14 $\mathrm{Nm}$ and after the application of the confidence factor the thresholds are $12 \mathrm{Nm}$ and $12 \mathrm{Nm}$. The interaction torques are bounded within the limits even in the presence of maximum stiffness.
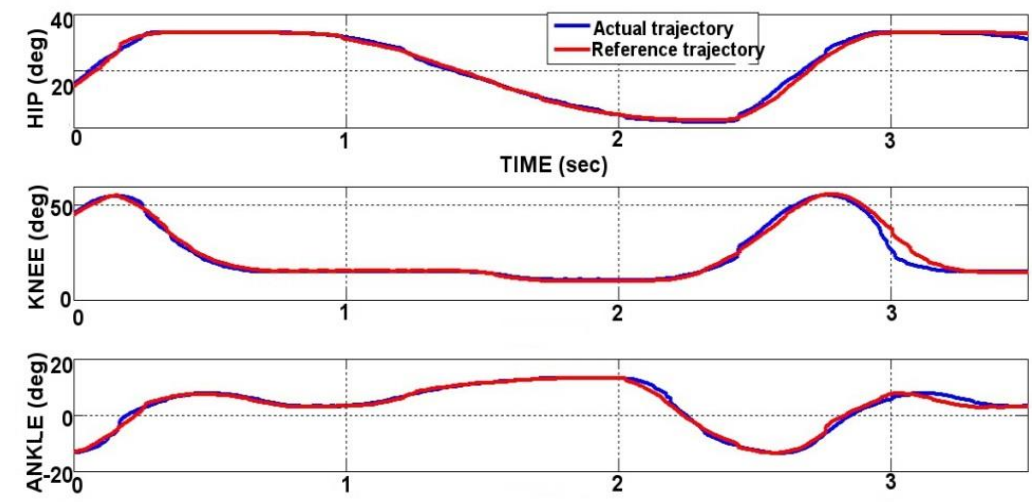

Fig. 8. Trajectory deviation of a healthy subject between trials 10 and 11

Since the flexion and extension movement of the knee joint is essential in walking for maintaining the transition between gait phases, the stiffness variation converges, after a few gait trials, as shown in fig 8 . The trajectory deviation is found to be within a small range, but with a delay, in the knee joint, as shown in fig 9. In case of the ankle joint the stiffness behavior shows a different pattern due to the compensation of ground reaction forces. In the ankle joint, the deviation from the reference position is found to be higher, which explains the pattern of stiffness variation.

The confidence factor is used to act on the joint gradually, by varying its stiffness. The consequence is the relax intervals that appear as negative slope (decreasing stiffness), which results in achieving a smooth behavior of the system. Lower confidence factors will result in few and shorter steps of stiffness variation, so the increment will be faster. On the contrary, a higher confidence factor will limit the increase of stiffness. The gradual increase in the stiffness value is due to the permanent difference in position error. The error in position of the joint in combination with the change in interaction torques results in a high stiffness value. 


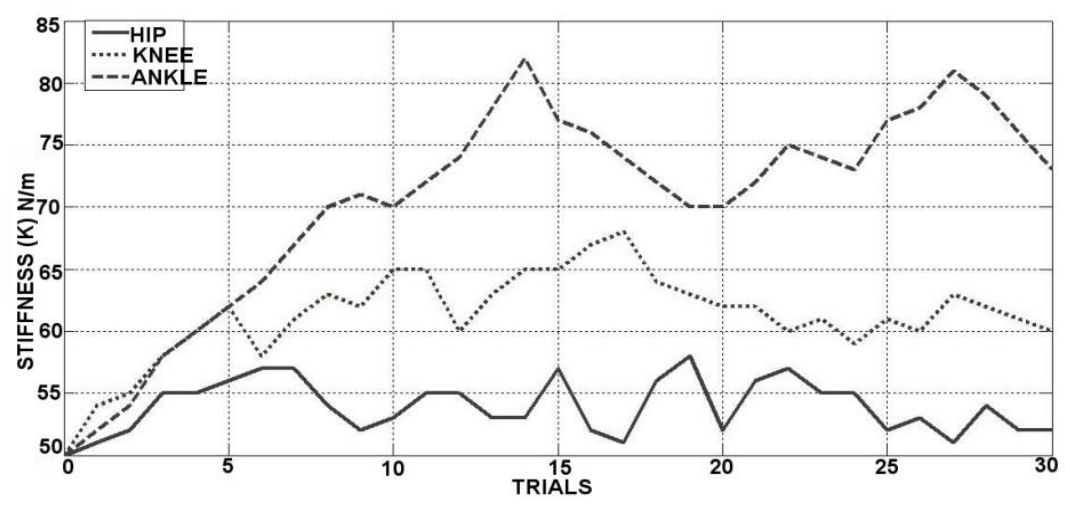

Fig. 9. Stiffness variation of a healthy subject

\section{Conclusion}

A user intention based adaptive walking strategy has been evaluated in function of the position error and human-orthosis interaction torques, thus ensuring an effective and safe therapy. The gait initiation is determined at the beginning of each gait cycle from the input orders received from the user. The stiffness value of each joint adapts dynamically to the user needs and keeps the position error bounded within the specified limits in real time. The wearable robot was tested with no body weight compensation, which demonstrates the reliability of the control strategy in terms of ensuring dynamic stability in presence of ground reaction forces. The results of the proposed control method were evaluated by comparing the resulting trajectory with a predefined gait trajectory. The experimental results showed that the user's gait intention was observed effectively with no delay and followed by the leg movement. The results demonstrated that the evolution of the stiffness value does not follow a similar pattern for all the joints. The stiffness value converges to be within a given range after a series of trials. The stiffness variation was in coordination with the flexion and extension movements. This demonstrates the efficiency of the proposed method for a real time process involving multiple joints.

This work has been the basis for a further study combining this gait initiation strategy with a brain machine interface that considers neurological signals and for the evaluation of the performance of the adaptive control in the assistance of incomplete SCI and Stroke patients, including the presence of muscle stimulation (FES), which, from the control perspective, acts as external disturbances.

\section{Acknowledgment}

This research is supported by project HYPER (Hybrid Neuroprosthetic and Neurorobotic devices for functional compensation and rehabilitation of motor disorders), grant 
CSD2009-00067 CONSOLIDER INGENIO 2010 from MINECO (Spanish Ministry for Science and Education)).

\section{References}

1. T. Yan, M. Cempini, C. M. Oddo and N. Vitiello, "Review of assistive strategies in powered lower-limb orthoses and exoskeletons," Robotics and Autonomous Systems, vol. 64, pp. 120-136, 2015.

2. L. Marchal-Crespo and D. Reinkensmeyer, "Review of control strategies for robotic movement training after neurologic injury," Journal of NeuroEngineering and Rehabilitation, vol. 6, no. 20, 2009.

3. D. Novak, et al"Automated detection of gait initiation and termination using wearable sensors," Medical Engineering and Physics, vol. 35, pp. 1713-1720, 2013.

4. W. Hasani, S. Mohammed, H. Rifaï and Y. Amirat, "Powered orthosis for lower limb movements assistance and rehabilitation," Control Engineering Practice, vol. 26, pp. 245 253,2014

5. T. Noda, et al,"Brain-Controlled Exoskeleton Robot for BMI Rehabilitation," in 12th IEEE RAS International Conference on Humanoid Robots, pp. 21-27, Osaka, Japan, 2012.

6. S. Wang, et al, "Design and Control of the MINDWALKER exoskeleton," IEEE Transactions on Neural Systems and Rehabilitation Engineering, vol. 23, no. 2, pp. 277 286, 2015.

7. H. Kawamoto, et al, "Voluntary motion support control of Robot suit HAL triggered by Bioelectrical signal for Hemiplegia," in 32nd International Conference of the IEEE EMBS, pp. 462-466, Buenos, Aires, 2010.

8. M. Aach, et al, "Voluntary driven exoskeleton as a new tool for rehabilitation in chronic spinal cord injury: a pilot study," The Spine Journal, vol. 14, pp. 2847-2853, 2014.

9. H. Kazerooni, J. L. Racine, L. Huang and R. Steger, "On the control of the Berkeley lower extremity exoskeleton (BLEEX)," in IEEE International Conference on Robotics and Automation, ICRA, pp.4353-4360, 2005.

10. W. van Dijk and H. van der Kooij, "XPED2: A passive exoskeleton with artificial tendons," IEEE Robotics and Automation Magazine, pp. 56-61, December 2014.

11. K. A. Strausser and H. Kazerooni, "The development and testing of a human machine interface for a mobile medical exoskeleton," in IEEE/RSJ International Conference on Intelligent Robots and Systems, pp. 4911-4916, San Francisco, CA, USA, 2011.

12. M. Talaty, A. Esquenazi and J. E. Briceño, "Differentiating ability in users of the ReWalk powered exoskeleton," in IEEE International Conference on Rehabilitation Robotics, Seattle, USA, pp.1-5, 2013.

13. M. Bortole, A. J. Del-Ama, E. Rocon, J. C. Moreno, F. Brunetti and J. L. Pons, "A Robotic Exoskeleton for Overground Gait Rehabilitation," in IEEE International Conference on Robotics and Automation, pp. 3356-3361, Karlsruhe, Germany, 2013.

14. V.Rajasekaran, J.Aranda and A.Casals, "Recovering planned trajectories in robotic rehabilitation therapies under the effect of disturbances," in International Journal of System Dynamics Applications, vol. 3, no.2, pp. 34-49, 2014.

15. V.Rajasekaran, J.Aranda, A.Casals and J.L.Pons "An Adaptive control strategy for postural stability using a wearable exoskeleton," in Robotics and Autonomous Systems, vol. 73, pp.16-23, 2015. 